\title{
Intraoperative radiotherapy for early breast cancer - insufficient evidence to change practice
}

\author{
Peter D. Sasieni@ and Elinor J. Sawyer
}

We argue that the evidence remains insufficient for use of intraoperative radiotherapy (IORT) in women with early stage breast cancer outside of a clinical trial, as the recently reported TARGIT-A trial does not provide sufficient evidence to conclude that IORT is superior to no radiotherapy.

Refers to Vaidya, J. S. et al. Long term survival and local control outcomes from single dose targeted intraoperative radiotherapy during lumpectomy (TARGIT-IORT) for early breast cancer: TARGIT-A randomised clinical trial. BMJ 370, m2836 (2020).

Despite results of a recent high-profile clinical trial, in which investigators concluded that risk-adapted targeted intraoperative radiotherapy (IORT) is an "effective alternative" to external beam radiotherapy (EBRT) (based on showing non-inferior 5-year local recurrence of $2.23 \%$ versus $1.02 \%$, respectively, difference $1.21 \%$, 95\% CI $0.33-2.09)^{1}$, we argue that the evidence remains insufficient for use of IORT in women with early stage breast cancer outside of a clinical trial. We see no reason to change the ASTRO consensus statement "low-energy X-ray IORT should continue to be used [only] within the context of a prospective registry or clinical trial to ensure long-term local control and toxicity outcomes are prospectively monitored" 2 .

The incidence of local recurrence following breast-conserving surgery (BCS) and EBRT has decreased considerably over the past 30 years. In a meta-analysis of randomized controlled trials that began before the year $2000,7.7 \%$ of women treated with BCS and radiotherapy had locoregional recurrence within 10 years of diagnosis ${ }^{3}$, compared with $25.1 \%$ in the BCS only arm. The addition of radiotherapy therefore resulted in a substantial absolute benefit (locoregional recurrence was avoided in $17.4 \%$ of women at 10 years) as well as a highly significant relative benefit (risk ratio $0.31 ; P<0.00001)$. When TARGIT-A was designed, the protocol assumed $6 \%$ of women in the EBRT arm would have local disease recurrence by 5 years ${ }^{4}$. However, the 5 -year local recurrence rate among women in the control arm (who received EBRT) was just under $1.0 \%$ - in TARGIT-A randomization took place either before ${ }^{1}$ or after $^{5}$ surgery with very similar risks observed in the control arms of both groups. This risk is similar to that observed in the PRIME II trial (2003-2009): $1.3 \%$ of women with low-risk early stage ER-positive, node-negative breast cancer (tumour diameter $\leq 3 \mathrm{~cm}$ ) had local relapse after BCS and EBRT 6 . This dramatic reduction in local recurrence over the years reflects remarkable progress in the treatment of early stage breast cancer.

In TARGIT-A a non-inferiority margin of $2.5 \%$ was adopted, implying that the investigators consider an additional 1 in 40 women having local recurrence within 5 years to be an acceptable outcome. This margin might have been reasonable considering that the 5 -year risk of local recurrence when the trial was designed was about $6 \%$ with EBRT and about $20 \%$ without radiotherapy, therefore, a treatment that was inferior by $2.5 \%$ would have a 5 -year risk of local recurrence of $8.5 \%$, which is considerably lower than with no radiotherapy. However, given the meta-analysis indicates a relative risk of about 0.3 for EBRT relative to no radiotherapy ${ }^{3}$ and given that 5 -year local recurrence in the EBRT arm of TARGIT-A was around 1\%, local recurrence without radiotherapy might have been around $3 \%$. In such circumstances, we argue that championing the superiority of IORT on the grounds that 5-year local recurrence has been shown to be $<3.5 \%$ is meaningless. Even if IORT is clinically noninferior to EBRT, no radiotherapy at all is also clinically noninferior, according to this definition. For example, in the PRIME II trial, after a median follow-up duration of 5 years, the ipsilateral breast tumour recurrence rate was $4.1 \%$ in women assigned to no radiotherapy versus $1.3 \%$ in those assigned to whole-breast radiotherapy: the absolute difference was only just greater than the $2.5 \%$ margin $^{6}$. Taking into account the imprecision of the estimated risk ratios, TARGIT-A does not provide sufficient evidence to conclude that IORT is superior to no radiotherapy and, in the absence of supportive evidence, IORT should not be used outside of clinical trials ${ }^{2}$.

Two randomized controlled trials have compared IORT with EBRT (ELIOT ${ }^{7}$ and TARGIT-A ${ }^{1,5}$ ), but none has compared IORT with no radiotherapy. In ELIOT, 35 patients in the IORT arm had local recurrence compared with four in the EBRT arm; at 5 years the risk was $4.4 \%$ versus $0.4 \%(P<0.0001)^{7}$. Data from TARGIT-A have been reported as two separate trials: one in women randomized prior to surgery ${ }^{1}$ and a second in women randomized post-surgery for whom a second operation was required to deliver IORT ${ }^{5}$. In the post-surgery cohort of TARGIT-A, 23 women in the IORT arm (4.0\%) had local recurrence at 5 years versus six $(1.0 \%)$ in the EBRT arm $(P=0.002)^{5}$. The report from the TARGIT-A pre-surgery cohort ${ }^{1}$ does not include $P$ values for local recurrence (they are presented for local-recurrence-free survival, an end point that pools all deaths with local recurrence), but sufficient data are provided to calculate these quantities. In the pre-surgery cohort, IORT is statistically inferior to EBRT in terms of local recurrence at 5 years (Kaplan-Meier estimates of local recurrence at five year years (intention-to-treat analysis) $2.23 \%$ versus $1.02 \%$ : risk difference $1.21 \%$, 95\% CI 0.33-2.09; $P=0.007$ calculated from the $95 \%$ CI provided) and after a median of 8.6 years $(60 / 1,140$ versus $24 / 1,158$; test of proportions $\left.\mathrm{X}^{2}=16.61 ; P<0.0001\right)$. Thus, both TARGIT-A cohorts demonstrate that IORT is in fact statistically inferior to EBRT.

\section{...both TARGIT-A cohorts} demonstrate that IORT is in fact statistically inferior to EBRT

Notwithstanding questions regarding the appropriateness of an informal meta-analysis of data from the three cohorts or of considering the pre-surgery TARGIT-A cohort in isolation, data from other randomized controlled cohorts fail to support the superiority of IORT over no radiotherapy. Data are also available from several single-arm trials of IORT, although these cannot provide evidence of superiority.

Among the TARGIT-A patients randomized pre-surgery ${ }^{1}$, EBRT was given in addition to IORT if post-surgery histopathological analysis suggested the patient had a high risk of local recurrence $(<1 \mathrm{~mm}$ tumour-free margin, $>25 \%$ in situ component, or lobular carcinoma; and at some centres the presence 
of a grade 3 tumour, positive lymph nodes or lymphovascular invasion). In an earlier report results are presented separately for those receiving IORT alone and those who receive IORT plus EBRT ${ }^{4}$. Those receiving EBRT in addition to IORT tended to have larger and higher-grade tumours and were more likely to have tumour-positive lymph nodes. These patients were more likely to die from breast cancer within 5 years of randomization $(8.0 \%$ versus $1.8 \%$ ) but despite their worse prognosis were less likely to have only local recurrence ( $0.9 \%$ versus $2.7 \%)$. Unfortunately, no data are presented to enable comparisons between IORT and EBRT in women whose post-surgery pathology suggests a low-risk of local recurrence (and who would therefore not have been offered EBRT had they already received IORT).

Evidence showing that IORT is better than no radiotherapy in women with early stage breast cancer is currently not available

Discriminating between women with good-prognosis breast cancer who nonetheless need radiotherapy and those who can safely avoid radiotherapy altogether remains a fundamental challenge. Even among women with pN0 cancer excised with clear margins, the 10-year risk of local recurrence depends on age (fivefold greater risk in women age $<40$ than in those $>70$ years of age), tumour grade (fourfold greater risk with high-grade relative to low-grade), tumour size and molecular subtype (ER-positive breast cancer confers a lower risk) ${ }^{3}$. Thus, some of the women enrolled in TARGIT-A are likely to have had a 10-year risk of recurrence with EBRT as low as $1 \%$ whereas for others it could have been as high as $12 \%$; furthermore, the absolute benefit of radiotherapy varies between $2 \%$ and $20 \%$ at 10 years. Indeed, this is what was observed in ELIOT $^{7}$. The 5-year risk of local recurrence in the IORT arm was 1.5\% among 452 women with no known risk factors but $11.3 \%$ among 199 with at least one poor prognostic factor (tumour diameter $>2 \mathrm{~cm}$, $\geq 4$ tumour-positive lymph nodes, grade 3 disease or triple-negative breast cancer $)^{7}$. Considering the relative risk of 2.2 in TARGIT-A, IORT would be clearly clinically inferior to EBRT in women who have a high risk of recurrence. However, women with none of these risk factors might opt to forgo radiotherapy because the additional benefits do not justify the inconvenience and toxicities ${ }^{6,8}$. This clinical dilemma is the rationale of PRIMETIME (ISRCTN41579286), in which women are stratified based on a risk score, IHC4 + C, that incorporates both immunohistochemical and clinical biomarkers: women with a very low risk $\mathrm{IHC} 4+\mathrm{C}$ score receive $\mathrm{BCS}$ alone whereas those with a low, medium or high score receive BCS plus radiotherapy. Other ongoing trials are also exploring the possibility of omitting radiotherapy in the treatment of women with a low risk of local recurrence: PROSPECT (ACTRN12610000810011), PRECISION (NCT02653755), IDEA (NCT02400190), LUMINA (NCT01791829), EUROPA (NCT04134598) and DBCG Natural (NCT03646955).

A question remains as to whether IORT might be a reasonable treatment for women with a low or medium risk of local recurrence or for women (such as current smokers) at a greater risk of cardiovascular disease or lung cancer: both of which are increased by historical EBRT regimens ${ }^{3}$. A three-arm trial comparing no radiotherapy to IORT and to EBRT in such women might be useful. With FAST-Forward showing that five fractions of $26 \mathrm{~Gy}$ radiotherapy are noninferior to the standard 15 fractions of $40 \mathrm{~Gy}$ in terms of local tumour control, toxicity and cosmesis ${ }^{9}$, whether the convenience and lower levels of radiation exposure associated with IORT justifies the inferior local recurrence, even if it were to be shown to be superior to no radiotherapy, needs to be considered.

The world is adopting five fractions ${ }^{9}$ as the standard of care approach, partly in response to COVID-19. Allowing that some women might opt not to receive radiotherapy at all (PRIME II ${ }^{6}$ and CALGB 9343 (REF. ${ }^{8}$ )), if radiotherapy is needed, short-course external beam approaches, not IORT, currently have the best level of evidence of effectiveness. Evidence showing that IORT is better than no radiotherapy in women with early stage breast cancer is currently not available. In such circumstances a drug therapy would never receive regulatory approval for use $\mathrm{e}^{10}$. Standards for radiotherapy should be no lower.

\section{Peter D. Sasieni (iD ${ }^{\bowtie}$ and Elinor J. Sawyer School of Cancer \& Pharmaceutical Sciences, King's College London, London, UK. \\ ${ }^{凶}$ e-mail: peter.sasieni@kcl.ac.uk https://doi.org/10.1038/s41571-020-00444-2}

1. Vaidya, J. S. et al. Long term survival and local control outcomes from single dose targeted intraoperative radiotherapy during lumpectomy (TARGIT-IORT) for early breast cancer: TARGIT-A randomised clinical trial. BMJ 370, m2836 (2020).

2. Correa, C. et al. Accelerated partial breast irradiation: executive summary for the update of an ASTRO evidence-based consensus statement. Pract. Radiat. Oncol. 7, 73-79 (2017).

3. Early Breast Cancer Trialists' Collaborative. et al. Effect of radiotherapy after breast-conserving surgery on 10-year recurrence and 15-year breast cancer death: meta-analysis of individual patient data for 10,801 women in 17 randomised trials. Lancet $\mathbf{3 7 8}$, 1707-1716 (2011).

4. Vaidya, J. S et al. An international randomised controlled trial to compare TARGeted Intraoperative radioTherapy (TARGIT) with conventional postoperative radiotherapy after breast-conserving surgery for women with early-stage breast cancer (the TARGIT-A trial). Health Technol. Assess. 20, 1-188 (2016).

5. Vaidya, J. S. et al. Effect of delayed targeted intraoperative radiotherapy vs whole-breast radiotherapy on local recurrence and survival: long-term results from the TARGIT-A randomized clinical trial in early breast cancer. JAMA Oncol. 6 , e200249 (2020).

6. Kunkler, I. H. et al. Breast-conserving surgery with or without irradiation in women aged 65 years or older with early breast cancer (PRIME II): a randomised controlled trial. Lancet Oncol. 16 266-273 (2015).

7. Veronesi, U. et al. Intraoperative radiotherapy versus external radiotherapy for early breast cancer (ELIOT) a randomised controlled equivalence trial. Lancet Oncol. 14, 1269-1277 (2013).

8. Hughes, K. S. et al. Lumpectomy plus tamoxifen with or without irradiation in women age 70 years or older with early breast cancer: long-term follow-up of CALGB 9343. J. Clin. Oncol. 31, 2382-2387 (2013).

9. Murray Brunt, A et al. Hypofractionated breast radiotherapy for 1 week versus 3 weeks (FASTForward): 5-year efficacy and late normal tissue effects results from a multicentre, non-inferiority, randomised, phase 3 trial. Lancet 395, 1613-1626 (2020).

10. Fleming, T. R. Current issues in non-inferiority trials. Stat. Med. 27, 317-332 (2008).

\section{Acknowledgements}

We would like to thank professors Jack Cuzick (biostatistics), Alastair Thompson (surgery) and Andrew Tutt (clinical radiotherapy) for discussions and suggestions in the preparation of this manuscript.

\section{Competing interests}

The authors declare no competing interests. 